\title{
Effect of the Characteristics of Solvent on the Shielding Effectiveness in Electromagnetic Shielding Materials
}

\author{
Qian Wei ${ }^{1, a}$ and Guohui Zhu ${ }^{2, b}$ \\ ${ }^{1}$ School of Metallurgical Engineering, Anhui University of Technology, \\ 59 Hudong Road, Maanshan, 243000, Anhui, China \\ ${ }^{2}$ School of Metallurgical Engineering, Anhui University of Technology, \\ 59 Hudong Road, Maanshan, 243000, Anhui, China \\ a18855581005@163.com, ${ }^{b}$ zhugh@ahut.edu.cn
}

Keywords: Electromagnetic shielding materials; Shielding efficiency; Surface resistance; Dielectric Constant.

\begin{abstract}
This essay analyses the experiment of xylene, ethyl acetate and acetone as solvent materials influencing the surface resistivity and electromagnetic shielding effectiveness of conductive filler composite material, as well as the main reasons for this difference from the perspective of material properties of the solvent. Analysis shows that, when the acrylic is the resin-based material, all three solutions can be used as a solvent to adjust the viscosity of the coating electromagnetic shielding. However, there is a huge difference in shielding effectiveness after spraying. This essay found that the dielectric constant has an important influence on the shielding effectiveness. While acetone is the solvent with the largest dielectric constant, the electromagnetic shielding effectiveness is worst. While xylene is a solvent, the electromagnetic shielding effectiveness is the best, and the volume fraction variation does not affect the electromagnetic shielding effectiveness. While ethyl acetate is a solvent, the influence is more complicated. There is need for a comprehensive analysis on the mobility and film-forming properties.
\end{abstract}

\section{Introduction}

With the innovation and development of electronic technology, more and more electrical industry and consumer electronics products are enhancing the vitality and efficiency of industrial production. People's life is undergoing a revolutionary change. However, when using electronic products, electromagnetic radiation released by electromagnetic waves from electronic components are inevitable. This presence of electromagnetic radiation has a huge impact on the environment[1]. The main appearance is that electromagnetic from electrical and electronic equipment affected the normal operation of the device itself[1], such as car safety[2], analysis and diagnosis of medical equipment[3] and so on. Electromagnetic radiation from digital transmission system can cause leakage of information[1], such as leaks from computer network data transfer process. Long-term electromagnetic exposure can cause fatigue of the body and induce a variety of tumours, posing a threat to human health[4]. Therefore, modern society has labelled electromagnetic radiation equivalent to the Modern Society "three wastes", called "fourth pollution" or "space pollution". Governments have developed the appropriate EMC (ElectroMagnetic Compatibility) standards against electromagnetic pollution for electrical and electronic equipment.

In order to meet EMC standards, electromagnetic shielding technology can be designed from the structural design of electronic products. But it often requires a lot of manpower and resources for research and development. So over the last decade, a lot of research work is done to study the development of high-performance electromagnetic shielding materials to achieve low cost and easy to use electromagnetic shielding technology. High-performance composite electromagnetic shielding materials is the one of the main directions for China's Ministry of Science and new materials research[5]. High-performance electromagnetic shielding materials is mainly composed of 
conductive filler electromagnetic shielding material. Its basic characteristics are, the use of a conductive component as a conductive body to build a network in the matrix material, the formation of the electromagnetic wave reflection loss and absorption loss by the conductive network to effectively reducing magnetic radiation, and to achieve the purpose of electromagnetic shielding[6]. Commonly used conductive components mainly are: conductive polymer material[7], metal powder[8], carbon nanotubes[9], and so on.

Currently, the research is focused on the influence of conductive component composition and volume fraction, and systematic study of the nature of the solvent on the electromagnetic shielding properties of the coating is still relatively small. In fact, the non-linear behaviour of conductive composites is usually a result of conductive mechanism and quantum mechanical tunnelling conductive paths and other synergistic results. When the conductive filler volume fraction is small, the conductive particles can not form a conductive path. Then this time only the quantum mechanical tunnelling effect is at work. When the conductive filler volume fraction is large, the conductive behaviour of the composite material is the result of the combination of conductive paths and tunnels. In this essay, as to the problems during experimental work, using nickel -based conductive component and an acrylic as resin-based material, we study and analyse the use of acetone, xylene and ethyl acetate as the solvent, the influence of different parameters on the electromagnetic shielding coating performance, and analyse the reasons of this impact.

\section{Experimental Materials and Methods}

Experiments are divided using pure nickel powder as a conductive component, using acrylic as resin matrix, using nickel powder as micron carbonyl nickel powder, and using acetone, xylene and ethyl acetate as the solvent. Nickel powder is first mixed with the nickel powder in accordance with an acrylic resin volume fraction of $15 \%$, and then added during the mixing volume fraction of $40 \%$ (calculated in the resin) to three solvents in order to facilitate even mixing. Stir the precursor into the ball mill for milling. Ball milled 90 minutes. Remove the pot from the milling, and then add in 5\%, $10 \%, 15 \%$ and $20 \%$ volume fraction with different solvents respectively. And stir into electromagnetic shielding paint. So the solvents from this study are of volume fraction of $45 \%, 50 \%$, $55 \%$ and $60 \%$.

According to People's Republic of China's requirements of national military standards (GJB 6190-2008), evenly sprayed the electromagnetic shielding paint spray on to a resin plate with a diameter of $115 \mathrm{~mm}$, to form an electromagnetic shielding coating after drying. Surface resistance was measured using a multimeter, with on average 5 sets of data measured on each sample. The shielding effectiveness of electromagnetic shielding coating was measured based on the national standards, using of PAN-L 5230C Agilent network analyzers and coaxial devices.

\section{Experimental Results and Analysis}

\section{Experimental Results}

Variation of electromagnetic in three different solvent volume fraction caused by changes in the surface resistance of the barrier coating is shown in Table 1 . Among which the $40 \%$ volume fraction is first added to the solvent in all samples (resin volume of $25 \mathrm{~mL}$ ), or $10 \mathrm{ml}$ of solvent for mixing powder and resin. After the numeric value plus the second column is prepared as a coating after adding volume fraction used to dilute paint for spraying.

Table 1 Surface resistance changes of electromagnetic shielding coating with different volume fraction of solvent

\begin{tabular}{ccc}
\hline Number & Solvent $(\mathrm{ml})$ & Surface resistance \\
\hline Acetone $\cdot 15$ & Acetone $10+5$ & 5.18 \\
Acetone $\cdot 20$ & Acetone $10+10$ & 5.72 \\
\hline
\end{tabular}




\begin{tabular}{ccc}
\hline Acetone $\cdot 25$ & Acetone $10+15$ & 5.08 \\
Acetone $\cdot 30$ & Acetone $10+20$ & 3.82 \\
Xylene $\cdot 15$ & Xylene $10+5$ & 3.48 \\
Xylene $\cdot 20$ & Xylene $10+10$ & 3.40 \\
Xylene $\cdot 25$ & Xyl ene $10+15$ & 4.06 \\
Xylene $\cdot 30$ & Xylene $10+20$ & 3.28 \\
& & 3.82 \\
Ethyl acetate $\cdot 15$ & Ethyl acetate $10+5$ & 5.58 \\
Ethyl acetate $\cdot 20$ & Ethyl acetate $10+10$ & 4.14 \\
Ethyl acetate $\cdot 25$ & Ethyl acetate $10+15$ & 3.72 \\
Ethyl acetate $\cdot 30$ & Ethyl acetate $10+20$ & \\
\hline
\end{tabular}

As seen from Table 1, for electromagnetic shielding coating layer composed of three different solvents, the surface resistance is of about the same order of magnitude with ohmic value between 3 to 6 ohms. But relatively speaking, seen from the surface resistance testing, overall, xylene has relatively minimal surface resistance. And with increasing amount of xylene, the surface resistance did not change significantly. When ethyl acetate was the solvent, the surface resistance increased in comparison with xylene. Even though with $20 \mathrm{ml}$ of the solvent, the surface resistance reached 5.58 ohms, but the overall effect is that when ethyl acetate was a solvent, a surface resistance was less than when acetone used as an electromagnetic shielding coating solvent. When using ethyl acetate and acetone as solvents, it has shown a trend of increasing surface resistance with increasing solvent content. From this point, the use xylene as solvent for electromagnetic shielding paint, with a relatively low surface resistance, with little effect on the volume of solvent, is easy to control the actual process. When using ethyl acetate or acetone as the solvent, although a smaller surface resistance can be achieved with a certain optimum solvent volume fraction, but as the volume fraction of the solvent changes, the surface resistance also showed a significant change. In the actual operation, it is required to maintain the optimum value, thus indicating certain difficulties.

Test results using three different solvents for the preparation of the electromagnetic shielding effectiveness of the barrier coating is shown in Figure 1-3. Seen from the figure, the solvents in three experiments can all achieve a relatively good electromagnetic shielding effectiveness of 40-80dB. Among which, when xylene was a solvent, electromagnetic shielding effectiveness reached 50dB or more. When ethyl acetate was the solvent, in addition to the volume fraction of ethyl acetate $20 \mathrm{ml}$, the volume fraction of the other shielding effectiveness all reached more than $50 \mathrm{~dB}$. For acetone as the solvent, electromagnetic shielding effectiveness reached to $40 \mathrm{~dB}$ and above. The above described electromagnetic shielding effectiveness of the test results are similar with the basic trends in surface resistance test, namely that the coating with lower surface resistance has better electromagnetic shielding effectiveness.

As seen from Figure 2, when using xylene as a solvent, as the volume fraction of xylene increase from $15 \mathrm{ml}$ to $30 \mathrm{ml}$, electromagnetic shielding effectiveness produced electromagnetic shielding coating has no significant change.That is, within the range of the volume fraction of the xylene solvent used in the experiments, xylene volume fraction cannot affect the electromagnetic shielding effectiveness of the coating. 


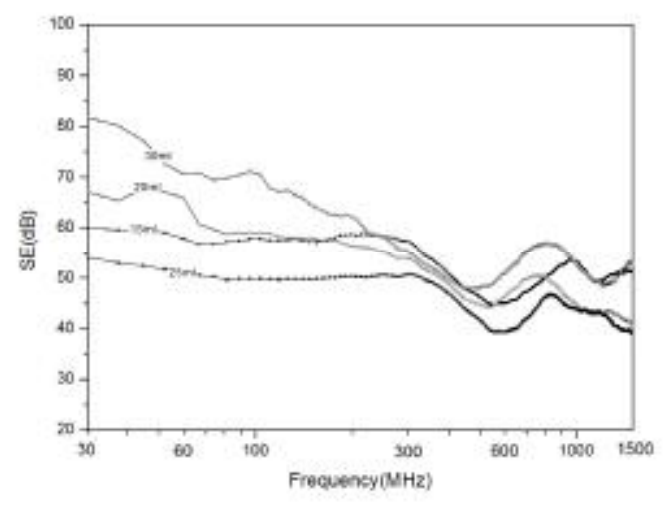

Fig.1 The electromagnetic shielding effectiveness with acetone as the solvent

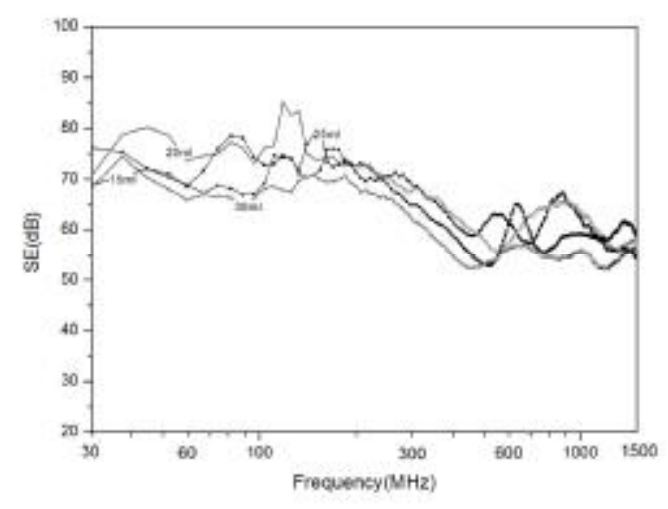

Fig. 2 The electromagnetic shielding effectiveness with xylene as the solvent

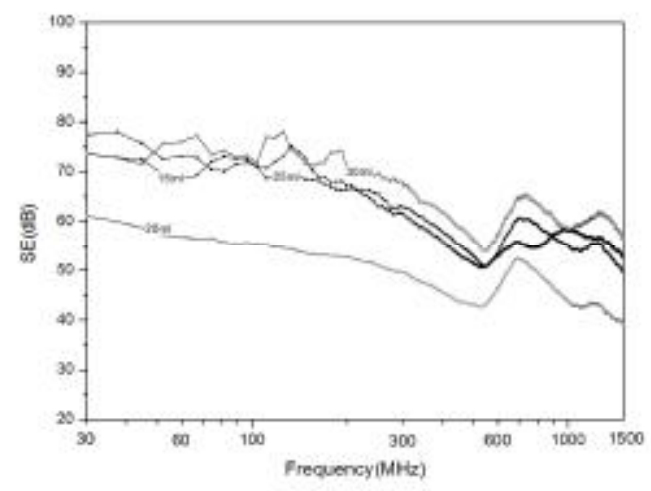

Fig.3 The electromagnetic shielding effectiveness with ethyl acetate as the solvent

When the solvent is ethyl acetate, as the volume fraction of the solvent increased from $15 \mathrm{ml}$ to $20 \mathrm{ml}$, the electromagnetic shielding effectiveness was significantly reduced. But when further increased of the volume fraction of the solvent, the electromagnetic shielding effectiveness of the coating came back to the original level. For when acetone was the solvent, in general, the electromagnetic shielding effectiveness of the coating is less than the use of xylene or ethyl acetate as solvent coating. With the increase of the volume fraction of acetone, from $15 \mathrm{ml}$ to $20 \mathrm{ml}$, the electromagnetic shielding effectiveness increases. But when the solvent is added to $25 \mathrm{ml}$, electromagnetic shielding effectiveness is decreased. When continue to $30 \mathrm{ml}$, electromagnetic shielding effectiveness increased again. It is noteworthy that, for the use of ethyl acetate and acetone as the solvent, although the coating electromagnetic shielding effectiveness changes with the volume fraction of the solvent, but the trend of the changes differ significantly. For the use of ethyl acetate as a solvent, in addition to $20 \mathrm{ml}$ volume fraction, the other three groups showed similar electromagnetic shielding effectiveness. However, when using acetone as the solvent, the change of electromagnetic shielding effectiveness is more complex. For example, when the volume fraction of the solvent increased from the time of $15 \mathrm{ml}$ to $20 \mathrm{ml}$, at low frequencies, the electromagnetic shielding effectiveness has increased. But in the high frequency, the electromagnetic shielding effectiveness decreased. And for different solvent volume fraction, there are significant differences in electromagnetic shielding effectiveness, which is similar to the overall trend of the experimental results.

\section{Experimental Analysis}

As for the effect of volume fraction of the solvent on the electromagnetic shielding effectiveness, this may be related to the characteristics of the solvent itself. This essay preliminary discusses the results 
from the solubility parameter of the solvent, the evaporation rate, surface tension and dielectric constant, etc. Basic properties of experiment reagents is shown in Table 2[10].

Table 2 Basic properties of experiment reagents

\begin{tabular}{ccccc}
\hline & $\begin{array}{c}\text { Solubility } \\
\text { Parameters } \\
\left(\text { Cal } / \mathrm{em}^{3}\right)^{1 / 2}\end{array}$ & $\begin{array}{c}\text { Relative Evaporation } \\
\text { Rate } \\
\text { (Methyl benzene }: 1)\end{array}$ & $\begin{array}{c}\text { Surface } \\
\text { Tension } \\
(\mathrm{mN} / \mathrm{m})\end{array}$ & $\begin{array}{c}\text { Dielectric } \\
\text { Constant }\end{array}$ \\
\hline Xylene & 8.8 & 0.34 & 30.69 & 2.3 \\
Ethyl acetate & 9.1 & 2.6 & 23.4 & 6.0 \\
Acet one & 9.9 & 4.8 & 23.7 & 20.7
\end{tabular}

It is seen from the table, for three solvents, the solubility parameters were similar. It is due to the fact that these three solvents can be dissolved in acrylic resin, and acrylic resin can used as an electromagnetic shielding substrate in paint solvents. For the conductive filler composite electromagnetic shielding materials, the basic principle is to use conductive component in the matrix structure as a conductive network, reflecting electromagnetic waves to reach the conductive plane. Among which the conductive network is constituted either by mutual contact between the conductive component particles, or the conductive particles may be in close proximity but without contact, by "tunnelling " and produce electro-conductive results[11]. Therefore, during the preparation of the electromagnetic shielding paint, the solvent's conductive component mobility and surface state will affect the structure of the conductive network.

The relatively large difference in three solvents is the "relative evaporation rate" and "dielectric constant". Among which the dielectric constant of the material of the electromagnetic wave can increase dielectric loss and improve the electromagnetic shielding effectiveness. But from the experimental results, however, xylene with the smallest dielectric constant has the best shielding effectiveness. This is due to the fact that the material used as the solvent in the coating layer mainly affect the process of forming a conductive network, and coating formation is done after the solvent was volatilised in the drying process. Therefore its electromagnetic properties does not significant effect the coating . But as the dielectric constant of the solvent separated ion has a greater capacity, it also has a strong solvating power[12]. It may make relative increased distance between the ion conductivity, affecting the electrical properties. Therefore, as acetone with the largest dielectric constant was a solvent, resulted in the least electromagnetic shielding effectiveness. Material's various relative evaporation rate plays an important role in the formation of conductive network. Acetone has a fast evaporation rate. It volatilises during the preparation of the coating, resulting in a decreased conductive matrix component fluidity, easy to form viscous component between the conductive resin film, resulting in a large increase of surface resistivity of conductive group resistance between the contact points and a lower electromagnetic shielding effects. From this analysis, increase the volume fraction of acetone will help to reduce the surface resistance and improve shielding effectiveness. Among which $25 \mathrm{ml}$ volume fraction of acetone as a solvent gave the minimum of electromagnetic shielding effectiveness. More research is necessary for the reason. Xylene has a lower evaporation rate, can act as a good solvent in the coating to facilitate the flowability conductive component. On the other hand xylene has a high surface tension, which means that maximum surface energy. It is difficult to form a dense film on surface of the conductive components. It is a good mutual contact of the conductive component and tunnelling. Xylene as a solvent gives a coating having minimal surface resistance and high electromagnetic shielding effects. Due to its low volatility when xylene was a solvent, xylene was added so as long as the volume fraction reaches conductive component liquidity requirements. Continue to increase xylene has little effect on surface resistivity and shielding effectiveness. When using ethyl acetate as the solvent, since the volatility of ethyl acetate is between acetone and xylene, therefore modest increase in ethyl acetate to increase the liquidity of the conductive component should help to improve the shielding effectiveness. But note that the relative low evaporation rate of ethyl acetate, and the surface tension is relatively small. So on 
one hand one needs to consider the impact of ethyl acetate conductive component liquidity, on the other hand needs to study at a appropriate viscosity, ethyl acetate resin may be combined to form the non-conductive film. Therefore, at low volume fraction of solvent, ethyl acetate mainly acts to increase fluidity of the conductive component from the diluted main substrate. The amount is not enough to form a dense film, thereby exhibiting high shielding effectiveness. When the volume fraction increases to $20 \mathrm{ml}$, then ethyl acetate substrate with a viscosity suitable for forming the film resulting in a decrease of shielding effectiveness. Thus further increasing in the content of ethyl acetate to dilute the effect of viscosity reduction, which leads to the detriment of the formation of a thin film, showing recovery of electromagnetic shielding effectiveness.

\section{Conclusions}

This essay analyses the experiment of xylene, ethyl acetate and acetone as solvent materials influencing the electromagnetic shielding effectiveness of conductive filler composite material. The results showd that the dielectric constant of solvent has an important influence on shielding effectiveness. Xylene as solvent with the best electromagnetic shielding effectiveness, however the change of volume fraction on the influence of the electromagnetic shielding effectiveness is not significant. So when xylene is a solvent, which has the largest dielectric constant, good electromagnetic properties and process performance are showd. Due to acetone has high relative evaporation rate, it won't be used as a solvent in electromagnetic shielding materials. While ethyl acetate is a solvent, the influence on coating on the shielding effectiveness of electromagnetic is more complex and it is need for a comprehensive analysis on the mobility and film-forming properties.

\section{References}

[1] Shijing Ding, Yuezhi Zhao. Research progress in electromagnetic shielding materials. Mater Rev, 2008,22(4):30(In Chinese)

[2] Di Wang.Research on protection effectiveness of vehicle electromagnetic shielding techniques in HEMP environment. Jilin:Jilin University, 2015(In Chinese)

[3] Yinbao Zhong, An Zhao, Yu Xiang. Protection technology of modern digital medical equipment under complicated electromagnetic environment.Chinese Medical Equipment Journal, 2011, 32(3):108(In Chinese)

[4] Yaoqiang Zhang, Yaodong Song, Xiaodong Liu et al. Electromagnetic pollution caused by high voltage over headlines and its harm to human healt. Elcctric Power Environmental Protection, 2012, 23(4): 33(In Chinese)

[5] Zhaoming Qu, Yisan Lei, Qingguo Wang et al. Design of high efficiency electromagnetic shielding composites and its shielding effectiveness test. High Voltage Engineering, 2012(In Chinese)

[6] Shacklette C. EMI Shielding measurements of conductive polymer blends. IEEE Instrumentation and Measurement Technology Conference, 1991:72-78

[7] Donghong Chen, Xinhai Yu, Xu Yongfen. Progress in research on electric conductive polymer materials. Chemistry and Adhesion,2012,34(6):61(In Chinese)

[8] Shipeng Ruan, Guohui Zhu, Weimin Mao. Effect of metal powder components on shielding effectiveness in shielding coating materials. Materials Review,2008,22(11):136(In Chinese)

[9] Kunhao Zhou, Xiaofang $\mathrm{Hu}$. Research progress in carbon nanotube filled polymer based composites for electromagnetic shielding. Chemical industry and engineering progress, 2012, 31(6): 1258(In Chinese)

[10] Ruibao Guan. Standards Manual of chemical reagent. Beijing: Standards Press of China, 2011(In Chinese)

[11] Rajagopal C , Satyam M.Studies on electrical conductivity of insulat or-conduct or composites. J Appl Phys, 1978, 49(11): 5536 
[12] Yueshan Wu, Yu Liya. Research on the concept of dielectric constant. Modern Electronics Technique, 2007, 2:177(In Chinese) 